\title{
Multiple Charging Station Location-Routing Problem with Time Window of Electric Vehicle
}

\author{
Wang Li-ying and Song Yuan-bin* \\ School of Naval Architecture, Ocean and Civil Engineering, Shanghai Jiao Tong University, Shanghai 200240, China
}

Received 19 August 2015; Accepted 11 November 2015

\begin{abstract}
This paper presents the electric vehicle (EV) multiple charging station location-routing problem with time window to optimize the routing plan of capacitated EVs and the strategy of charging stations. In particular, the strategy of charging stations includes both infrastructure-type selection and station location decisions. The problem accounts for two critical constraints in logistic practice: the vehicle loading capacity and the customer time windows. A hybrid heuristic that incorporates an adaptive variable neighborhood search (AVNS) with the tabu search algorithm for intensification was developed to address the problem. The specialized neighborhood structures and the selection methods of charging station used in the shaking step of AVNS were proposed. In contrast to the commercial solver CPLEX, experimental results on small-scale test instances demonstrate that the algorithm can find nearly optimal solutions on small-scale instances. The results on large-scale instances also show the effectiveness of the algorithm.
\end{abstract}

Keywords: Electric vehicles, Vehicle routings, Location-routing problem, Green logistics, Hybrid metaheuristics

\section{Introduction}

With the increasing worldwide concern on the environment, the negative effects of logistic operations, particularly in terms of greenhouse gas emission, can no longer be neglected. Electric vehicles (EVs), powered by the reproducible resource, are currently considered to be a crucial alternative to conventional internal combustion vehicles (ICVs) to improve the sustainability of logistic operation. In contrast to ICVs, EVs have both environmental and economical advantages [1]. Several industries have started to use EVs for distribution operation in recent years, such as the consumer goods industry [2,3]. In addition, the logistic giant, DHL, has engaged more than 304 EVs for delivery activities and plans to adopt $140 \mathrm{EVs}$ for delivery tasks in Bonn/Germany by 2016 [4].

In planning last-mile delivery task with EVs, the potential requirement of charging en route resulting from limited battery capacity should be rendered. Given the deficiency of public charging stations and the requests of logistic operations, such as time-definite deliveries, it's regarded as a promising alternative that the logistic enterprise operates its own charging stations [6].

Several types of charging infrastructure exist in practice, and each has its pros and cons. For example, although the battery swap station (BSS) can provide faster service than other types of charging stations, the establishment cost of BSS is much higher than that of others [5]. The infrastructure-type decision of a station has a great effect on

* E-mail address: ybsong@sjtu.edu.cn ISSN: 1791-2377 @ 2015 Kavala Institute of Technology. All rights reserved. the location decision of the station and the routing plan of EVs, while it is swayed by the location of the station and routing plan. The location of the charging station also has a great influence on the routing plan of EVs, and the routing plan of EVs is a key factor to locate charging stations [6]. Thus, these three problems should be addressed simultaneously to obtain an optimal solution. Two constraints of last-mile delivery practice, that is, customer time windows and vehicle loading capacity, are also critical for the routing plan and should be incorporated into the problem [7]. The time window constraint is quite interesting because the recharging time for an $\mathrm{EV}$ is determined by both the charging infrastructure and the distance traveled from the last charging station, and the arrival time at the customer after a visit is affected by the charging time in the preceding station and is restricted by the time window.

This study introduces the EV multiple charging station location-routing problem with time window (EV-MCSLRPTW). The problem incorporates the location decision and the type selection of charging infrastructure with the vehicle-routing plan, and considers customer time windows and the loading and battery capacities of vehicles. Each type of charging infrastructure is associated with a particular construction cost and charging rate. The problem is intended to provide an optimal solution with a minimal cost for the logistic enterprise that plans to adopt EVs for distribution and to construct its own charging stations. The problem minimizes the total cost, including the construction cost of the charging station, the charging cost of EVs, and the labor cost of drivers. Given that the problem extends the locationrouting problem (LRP), exact solution methods are incapable of addressing realistically sized instances within reasonable time periods [8]. Thus, we develop a hybrid heuristic named adaptive variable neighborhood search (AVNS)/tabu search (TS) to solve the problem, which 
integrates an AVNS heuristic with the TS algorithm for intensification. The AVNS algorithm incorporates the adaptive idea from the adaptive large neighborhood search (ALNS) into the variable neighborhood search (VNS) framework to bias the random shaking phase of VNS. The performance of AVNS/TS is tested on several sets of instances in numerical studies. Experiments on small-scale instances are used to evaluate the solution quality of AVNS/TS, and tests on large-scale instances are adopted to assess the effectiveness of the algorithm.

The remainder of this paper is organized as follows: Section 2 briefly reviews the literature related to EV-MCSLRPTW. Section 3 illustrates the mathematical formulation of the problem. Section 4 provides the solution approach. Section 5 demonstrates the parameter setting, the generation of new E-MCS-LRPTW instances, and the numerical studies. Finally, Section 6 concludes the study and summarizes the future works.

\section{Related background}

In this section, we review the literature related to EV-MCSLRPTW. Two streams of previous studies are most relevant to the present research. One is the LRP, and the other is the routing planning of alternative fuel vehicles (AFVs).

\subsection{Location routing problem}

A network is normally represented by a graph that is composed of a set of nodes and edges. The task of network clustering is to divide a network into different clusters based on certain principles. Each cluster is called a community. The LRP combines two classical planning tasks in logistics, that is, optimally locating depots and planning vehicle routes from these depots to geographically scattered customers [8]. These two interdependent problems have been addressed separately for a long time, which often leads to suboptimal planning results. The idea of LRP started in the 1960s, when the interdependence of the two problems was pointed out $[9,10]$. The variants of the LRP have been frequently studied in recent years. Such variants include the capacitated LRP (CLRP) with constraints on depots and vehicles [20,21], the LRP with multi-echelon of networks $[11,12]$, the LRP with inventory management $[13,14]$, and the LRP with service time windows [15-17]. For the variant problem with time windows, Semet and Taillard incorporated the time window constraint to the LRP for a special case of the road-trainrouting problem [15]. Zarandi et al. studied the CLRP with fuzzy travel time and customer time windows, in which a fuzzy chance-constrained mathematical program was used to model the problem [16]. Later, they extended the problem by adding the fuzzy demands of customers and developed a cluster-first route-second heuristic to solve the problem [17]. A detailed review of the LRP variants can be found in two recent surveys $[18,19]$.

For the solution method of LRP, studies that focus more on heuristics than on exact methods have been observed in the existing literature probably, because the LRP combines two nondeterministic polynomial-time-hard problems. Most of the heuristic methods can be classified into two categories. One category commonly applies modified metaheuristics to improve the initial solution generated in a previous step. For instance, to solve the CLRP, Jokar and Sahraeian first applied a greedy approach to produce an initial solution and then proposed the simulated annealing (SA) algorithm to exploit better solutions from the initial solution [20].
Hemmelymay et al. applied their ALNS metaheuristic enhanced by a local search algorithm in improving the initial solution to address the CLRP [21]. The other category is executed first by separating the LRP into two subproblems - the vehicle-routing problem (VRP) and the facility location problem - and then by solving the subproblems sequentially or iteratively. Perl and Daskin first proposed the idea of iterating between locational and routing phases, and the idea has been improved in several studies later [22].

\subsection{Routing problem of alternative fuel vehicles}

The second strand of the relevant literature consists of the routing problems that consider the limited driving range of vehicles and the possibility of refueling en route. Conrad and Figliozzi introduced the recharging VRP, in which vehicles with a limited range are allowed to recharge at certain customer stations within a fixed time [23]. Erdogan and Miller-Hookers presented the green VRP (G-VRP) for routing AFVs and solved the problem with two algorithms. In G-VRP, refueling stations are assumed to be independent of customer sites, and an AFV may refuel at these stations within a fixed time [24]. Later, Schneider et al. incorporated the time window constraint into the G-VRP and proposed the EV-routing problem with time windows and recharging station (E-VRPTW) [7]. The charging time in E-VRPTW is not fixed but instead is related to the battery charge of an EV upon arrival at the station. To address the problem, they developed a hybrid heuristic that combines the VNS with the TS algorithm (VNS/TS). Schneider et al. then introduced the VRP with intermediate stops (VRPIS), which generalized the G-VRP, and solved the problem by AVNS [29]. Five route selection methods and three vertex sequence selection methods were utilized in the adaptive shaking phase of AVNS. Felipe et al. proposed several heuristics to address the G-VRP with multiple technologies and partial recharges. The problem extends the G-VRP by incorporating different technologies for battery recharge and the possibility of partial recharges [25]. Goeke and Schneider combined the EVRPTW with a mixed fleet of EVs and ICVs, and utilized realistic energy consumption functions in their problem [26]. The resulting problem was solved by an ALNS with a local search for intensification. Yang and Sun adopted the simultaneous optimization idea from the LRP to the context of $\mathrm{EV}$ and proposed the BSS location-routing problem of EVs [6]. The problem is intended to minimize infrastructure and shipping costs by determining the station location and vehicle-routing plan jointly under a driving range limitation. For the solution method, they employed the concept of solving separate sub-problems iteratively from the LRP and proposed two hybrid heuristics [22]. In detail, one algorithm called TS-modified Clarke-Wright saving (MCWS) combines the TS algorithm for location strategy and the MCWS method for the routing decision. The other approach named SIGALNS includes four main phases: initialization, location sub-problem, routing sub-problem, and improvement. Iterative greedy (IG) is utilized in the location phase, and an ALNS in the routing phase.

Most of the existing studies on logistic operation with EVs focus on the routing plan of vehicles with fixed charging stations. However, for a logistic company that plans to construct its own charging stations to support distribution tasks, the strategy of charging stations and routing plan are both critical to obtain an optimal solution. To the best of our knowledge, the problem studied by Yang et al. is the closest to the EV-MCS-LRPTW defined in the 
present study. However, several fundamental differences exist between the two problems. First, their problem considers BSS to be the only type of charging infrastructure, whereas several types of infrastructure with distinct charging rates, construction costs, and electricity prices are regarded in the present research. The type selection of infrastructure is also optimized, along with the location decision and routing plan in our problem. Previous studies have implied that, aside from BSS, other types of charging infrastructure are frequently utilized in practice [5,25]. As illustrated before, each type of charging station has its cons and pros. Thus, the type selection is worthwhile to incorporate into the problem. Furthermore, the EV-MCS-LRPTW incorporates a critical constraint in real-world practice, the service time window, which has a strong effect on both the routing plan and type selection. Given that various types of stations are allowed in this work, the components considered in the objective function of EV-MCS-LRPTW are different from those in their work. Instead of shipping cost, driver wage and charging cost are tackled with the construction cost in the objective function evaluated in this study.

\section{Mathematical model}

The EV-MCS-LRPTW is defined in a complete, directed graph $G=(V, A)$. Set $C$ denotes the set of customers, and set $R$ denotes the set of candidate charging station sites. In particular, when a candidate station is at a customer site, the candidate station is treated as a dummy vertex of the customer site. The dummy vertex belongs to the set of candidate station sites, $R$, but shares the same location features as the customer. One single depot is considered in this problem and is denoted by vertices $O$ and $o^{\prime}$, where all the routes start from $o$ and end at $o^{\prime}$. Set $V$ consists of sets $C, R$, and both instances of the depot. The set of arcs is given by $A=\{(i, j) \mid i, j \in V, i \neq j\}$. A charging station is assumed to be located at the depot, in which each EV should be fully charged to battery capacity $Q$ when it returns for fairness of comparison.

Each customer vertex $i$ is characterized by a nonnegative demand $q_{i}$, a fixed service time $s_{i}$, and a time window $\left[e_{i}, l_{i}\right]$. The demand $q_{i}$ is assumed to be within the loading capacity $U$. Each candidate site $i \in R$ is provided with $\Lambda$ types of charging stations as options. Each infrastructure type $r \in \Lambda$ is associated with the construction cost $c_{i}^{r}$, constant charging rate $\lambda^{r}$, and electricity price $c_{e}^{r}$. The construction cost $c_{i}^{r}$ is determined by both type and location. Upon each visit to a station site $i \in R$, a vehicle $k$ is assumed to be fully charged with the charging time $\theta_{i}^{k}$. The charging time $\theta_{i}^{k}$ is based on both the charging rate and the charge level of the vehicle upon arrival at the station. Each arc is associated with distance $d_{i j}$ and travel time $t_{i j}$. The electricity consumption rate of a vehicle is assumed to be a constant $\varepsilon$. A fleet of identical EVs is considered in this work.

EV-MCS-LRPTW is formulated as a mixed-integer programming model. The binary variable $y_{i}^{r}$ defines whether or not to locate a type $r$ charging station at a candidate site $i \in R$. For every arc $(i, j) \in A$, the binary decision variable $x_{i j}^{k}$ takes the value of 1 when an arc $(i, j) \in A$ is traveled by vehicle $k$ and takes the value of 0 otherwise. The variable $\tau_{i}^{k}$ denotes the arrival time, $u_{i}^{k}$ denotes the remaining load, and $f_{i a}^{k}$ denotes the remaining charge level of vehicle $k$ upon arrival at vertex $i \in V$. The variable $f_{i}^{k}$ specifies the charging amount of vehicle $k$ at vertex $i \in R$.

Given the definitions of parameters and variables, the mathematical model is defined as follows:

Minimize

$$
f=\sum_{i \in R \cup\left\{o^{\prime}\right\}} \sum_{r \in \Lambda} c_{i}^{r} \cdot y_{i}^{r}+\sum_{k \in K} \sum_{i \in R \cup\left\{o^{\prime}\right\}} \sum_{r \in \Lambda} f_{i}^{k} \cdot c_{e}^{r} \cdot y_{i}^{r}+c_{d} \cdot \sum_{k \in K} \tau_{o^{\prime}}^{k}
$$

Subject to

$\sum_{j \in V /\left\{o^{\prime}\right\}, j \neq i} \sum_{k \in K} x_{j i}^{k}=1 \quad \forall i \in C$

$\sum_{j \in V \backslash\{0\}, i \neq j} x_{i j}^{k}-\sum_{j \in V \backslash\{0\}, i \neq j} x_{j i}^{k}=0$ $\forall i \in V \backslash\left\{o, o^{\prime}\right\}, \forall k \in K$

$\sum_{j \in V \backslash\{0\}} x_{o j}^{k}=1 \quad \forall k \in K$

$\sum_{j \in V \backslash\{0\}} x_{o j}^{k}-\sum_{j \in V \backslash\left\{o^{\prime}\right\}} x_{j o^{\prime}}^{k}=0 \quad \forall k \in K$

$U \cdot\left(x_{i j}^{k}-1\right) \leq u_{j}^{k}-u_{i}^{k}+q_{i} \cdot x_{i j}^{k} \leq U \cdot\left(1-x_{i j}^{k}\right)$ $\forall i \in V \backslash\left\{o^{\prime}\right\}, \forall j \in V \backslash\{o\}, i \neq j, \forall k \in K$

$u_{i}^{k} \geq 0 \quad \forall i \in V \backslash\left\{o^{\prime}\right\}, \forall k \in K$

$u_{0}^{k} \leq U \quad \forall k \in K$

$Q \cdot\left(x_{i j}^{k}-1\right) \leq f_{j a}^{k}-\left(f_{i a}^{k}+f_{i}^{k}\right)+\varepsilon \cdot d_{i j} \cdot x_{i j}^{k} \leq Q \cdot\left(1-x_{i j}^{k}\right)$ $\forall i \in V \backslash\left\{o^{\prime}\right\}, \forall j \in V \backslash\{o\}, i \neq j, \forall k \in K$

$f_{o a}^{k}=Q \quad \forall k \in K$

$f_{i}^{k}=\left(Q-f_{i a}^{k}\right) \cdot \sum_{r \in \Lambda}\left(y_{i}^{r}\right) \quad \forall i \in R \mathrm{U}\left\{o^{\prime}\right\}, \forall k \in K$

$\sum_{r \in \Lambda} y_{i}^{r} \leq 1 \quad \forall i \in R U\left\{o^{\prime}\right\}$

$f_{i}^{k}=\theta_{i}^{k} \cdot \sum_{r \in \Lambda}\left(y_{i}^{r} \cdot \lambda^{r}\right) \quad \forall i \in R U\left\{o^{\prime}\right\}, \forall k \in K$

$f_{i}^{k}=0 \quad \forall i \in C, \forall k \in K$

$f_{i a}^{k} \geq 0 \quad \forall i \in V, \forall k \in K$

$\tau_{i}^{k}+t_{i j} \cdot x_{i j}^{k}+s_{i} \cdot x_{i j}^{k}-\tau_{i}^{k} \cdot\left(1-x_{i j}^{k}\right) \leq \tau_{j}^{k}$ $\forall i \in C \mathrm{U}\{o\}, \forall j \in V \backslash\{o\}, \forall k \in K$

$\tau_{i}^{k}+t_{i j} \cdot x_{i j}^{k}+\theta_{i} \cdot x_{i j}^{k}-\tau_{i}^{k} \cdot\left(1-x_{i j}^{k}\right) \leq \tau_{j}^{k}$ 


$$
\forall i \in R, \forall j \in V \backslash\{o\}, \forall k \in K
$$

$e_{i} \leq \tau_{i}^{k} \leq l_{i} \quad \forall i \in V \mathrm{U}\left\{o^{\prime}, o\right\}, \forall k \in K$

$y_{i}^{r} \in\{0,1\} \quad \forall i \in R, \forall r \in \Lambda$

$x_{i j}^{k} \in\{0,1\} \quad \forall i \in V \backslash\left\{o^{\prime}\right\}, \forall j \in V \backslash\{o\}, i \neq j, \forall k \in K$

$f_{i}^{k} \geq 0 \quad \forall i \in V, \forall k \in K$

Objective function (1) minimizes a mixed cost, where the first term denotes the construction cost of charging stations, the second term the cost of electricity recharged at depot and stations, and the third term the diver wage associated with working time. Constraints (2) guarantee that each customer is visited exactly once. Constraints (3) ensure that the number of incoming arcs is equal to that of outgoing arcs for each vertex, except for the instances of the depot. Constraints (4) and (5) ensure that all the employed vehicles start and end routes at the depot. Constraints (6) and (7) enforce the fulfillment of demands at customer nodes, and Constraints (8) restrict the initial cargo load level of a vehicle to its capacity. Constraints (9) link the battery levels of the vehicle at the vertices $i$ and $j$ of a traveled arc $(i, j)$. Both Constraints (6) and (9) adopt the idea from the big-M method. Constraints (10) and (11) ensure that each vehicle leaves the depot or a located station with a fully charged battery. Constraints (12) confine the type of infrastructure located at a candidate site to be one. Constraints (13) define the simplified relationship between charging time and charging amount at located charging stations. Constraints (14) prevent a vehicle from charging at a vertex from a customer set. Constraints (15) guarantee that each vehicle has sufficient power to reach a located station or depot. Constraints (16) and (17) establish the relation between arrival times at vertices $i$ and $j$ if the $\operatorname{arc}(i, j)$ is traveled. Constraints (17) particularly cover the condition, where the arc $(i, j)$ starts with a charging station. Constraints (18) ensure that all customer vertices are visited within their time windows. Constraints (19) to (21) define the natural features of the variables.

\section{Proposed method for EV-MCS-LRPTW}

In this section, a hybrid heuristic that integrates AVNS with TS for intensification is proposed to address the problem. The approach is inspired by VNS/TS, a combination of VNS and TS, which has demonstrated its quality on several routing problems [7,27]. AVNS, which was proposed by Stenger et al. in 2012, is an approach that integrates the adaptive concept from ALNS into the route and customer selection in the shaking phase of VNS. AVNS has successfully been applied in several combinatorial optimization problems, including the multi-depot VRP with private fleet and common carriers, and the VRPIS [28,29]. TS is a renowned metaheuristic that guides a local search to explore the solution space beyond local optimality [30]. The approach is often associated with diversification and intensification mechanisms to obtain effective algorithms.

Algorithm 1 Pseudocode of the AVNS/TS.

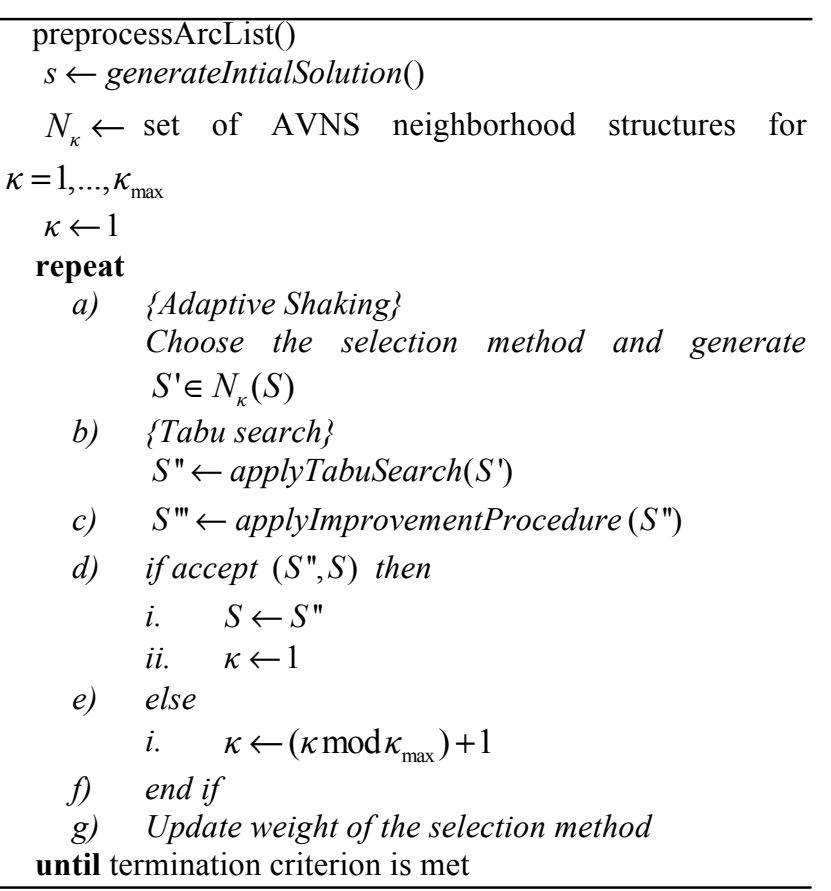

Algorithm 1 presents an overview of AVNS/TS in pseudocode. The algorithm starts with a series of preprocessing procedures (Section 4.1). An initial solution $S_{0}$ with a given number of vehicles is then generated (Section 4.3). Infeasible solutions are allowed during the search, and a penalty mechanism is applied to measure the violations (Section 4.2). The initial solution is iteratively improved by AVNS, TS, and an improvement procedure. In detail, the AVNS is mainly used to diversify the search based on the predefined neighborhood structures (Section 4.4). The solution from the AVNS phase, $S^{\prime}$, is then served as an input and processed in the TS step (Section 4.5). An improvement procedure is finally executed on the solution, $S^{\prime \prime}$, from TS to find an advanced one, (Section 4.6). The solution $S^{\prime \prime \prime}$ is eventually evaluated by an acceptance criterion based on SA (Section 4.7).

\subsection{Preprocessing procedure}

In the first step, the preprocessing procedure removes the infeasible arcs. An arc $(v, w)$ is regarded as infeasible if one of the conditions defined in Eqs. (22) to (24) holds. In detail, Eq. (22) addresses the violation of loading capacity, and Eqs. (23) and (24) address the violation of time windows [31].

$$
\begin{aligned}
& v, w \in V \wedge q_{v}+q_{w}>U \\
& v \in V \mathrm{U}\{o\}, w \in V \mathrm{U}\left\{o^{\prime}\right\} \wedge e_{v}+t_{v}^{s}+t_{v w}>l_{w} \\
& v \in V \mathrm{U}\{o\}, w \in V \wedge e_{v}+t_{v}^{s}+t_{v w}>l_{w}
\end{aligned}
$$

Given that each candidate site is assigned multiple types of infrastructure for selection, the second step of the procedure, called station vertex preprocessing, splits each candidate station site into various dummy vertices to simplify the calculation. Figure 1 gives a graphical illustration of the process. Before this preprocessing step, a candidate station site $i$ is associated with $\Lambda$ types of charging infrastructures, in which each type has its distinct construction cost, charging rate, and electricity price. After the procedure, the candidate site $i$ is replaced with $\Lambda$ 
dummy vertices, as shown in the lower part of the figure, where each dummy vertex denotes a station site with only one type of infrastructure in the same place as the vertex $i$.

\section{Before station vertex preprocessing}

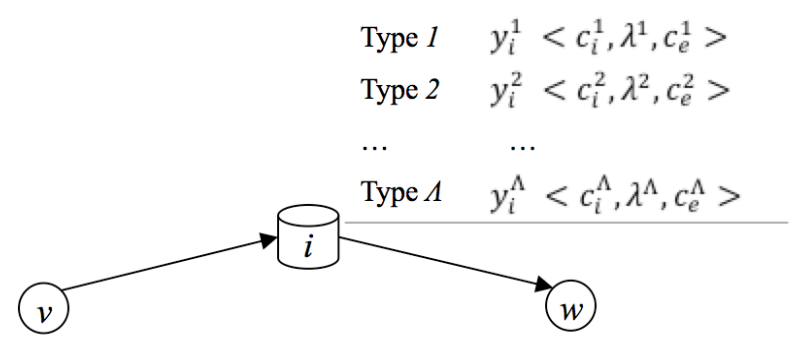

After station vertex preprocessing

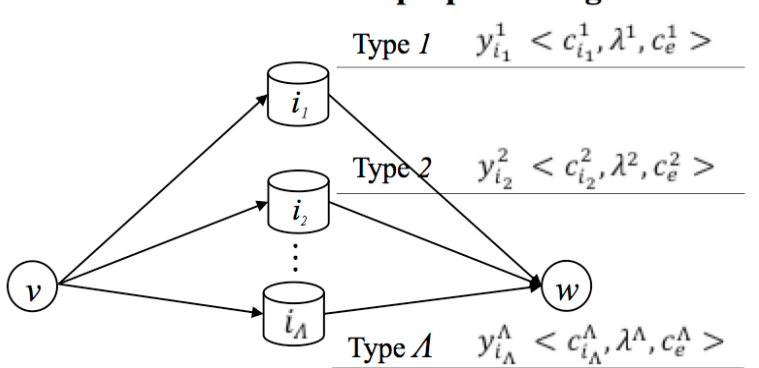

Fig. 1. Graphical illustration of station vertex preprocessing

\subsection{Penalty calculation}

The AVNS/TS algorithm allows the violation of certain constraints during the search. The violation is reflected as the penalized cost in a generalized objective function. This section introduces the penalized cost generated from the violations of loading capacity, battery capacity, and time window. Let a sequence of vertices $\left\langle v_{0}, v_{1}, \ldots, v_{n}, v_{n+1}\right\rangle$ represent a route $r$, where vertices $v_{0}$ and $v_{n+1}$ denote the instances of depot. The routing plan of solution $S$ can be presented as $S=\left\{r_{k}, k=1,2, \ldots, m\right\}$. Then the capacity violation of a route $r$ can be calculated as

$$
L_{\text {cap }}(r)=\max \left\{\sum_{i=0}^{n+1} q_{v_{i}}-C, 0\right\}
$$

The total capacity penalty of a solution is computed by summing up the violations of all routes:

$$
L_{\text {cap }}(S)=\sum_{i=1}^{m} L_{\text {cap }}\left(r_{k}\right)
$$

Similar to the approach in E-VRPTW, the variable $\Upsilon_{v_{i}}^{\rightarrow}$ is defined to track the electricity usage of a vehicle at each vertex of its route $r=\left\langle v_{0}, v_{1}, \ldots, v_{n}, v_{n+1}\right\rangle$ to compute battery capacity violation [7]. $\Upsilon_{v_{i}}$ records the accumulated electricity consumption from the last charging station or depot (see Eq. (27)). The variable $P_{v_{i}}$ represents the feasibility state of the vehicle at each vertex. If a vehicle violates the battery capacity $Q$ at vertex $v_{i}$, then $P_{v_{i}}$ is set as the excess electricity amount at that vertex; otherwise, $P_{v_{i}}$ is equal to 0 (see Eq. (28)).

$$
\Upsilon_{v_{i}}^{\rightarrow}=\left\{\begin{array}{ccc}
\mathcal{E} \cdot d_{v_{i-1} v_{i}} & \text { if } \quad v_{i-1} \in R \\
\Upsilon_{v_{i-1}}^{\rightarrow}+\mathcal{E} \cdot d_{v_{i-1} v_{i}} & \text { if } & v_{i-1} \in V / R
\end{array} \quad i \in\{1,2, \ldots, n+1\}\right.
$$

$$
P_{v_{i}}=\max \left\{\mathfrak{\Upsilon}_{v_{i}}^{\rightarrow}-Q, 0\right\} \quad i \in\{1,2, \ldots, n+1\}
$$

Given the definition of $P_{v_{i}}$, the battery capacity violation of a route $r$ is calculated by summing up the vehicle excess consumption amount at every station en route and upon return to the depot (see Eq. (29)). The battery capacity penalty of a solution $S$ is then presented as the total of the violations of all routes (see Eq. (30)).

$$
\begin{aligned}
& L_{b a t}(r)=\sum_{v_{i} \in R \cup\left\{0^{0}\right\}} P_{v_{i}} \\
& L_{b a t}(\mathrm{~S})=\sum_{k=1}^{m} L_{b a t}\left(r_{k}\right)
\end{aligned}
$$

In this work, the time window is calculated based on the methodology developed by Nagata et al. and enhanced by Schneider et al. [32,33]. The principle of the approach is to count the penalty on the vertex where the violation occurs, instead of propagating the violation along the entire route. For the consecutive vertices of that vertex, the approach assumes that the vehicle can travel back in time and start service at the latest feasible moment in the violation vertex.

In the VRPTW, the approach can calculate the time window penalty $L_{t w}(r)$ for conventional inter-route moves at constant time. However, given that the charging time of a vehicle is related to the traveled distance, recalculation is necessary in certain situations. As in the E-VRPTW by Schneider and Stenger, we adopt the methodology with modification in this work [7]. Let $r=\left\langle v_{0}, \ldots, u, v, w, \ldots, v_{n+1}\right\rangle$ be a route that is generated by combining the two partial routes $\left\langle v_{0}, \ldots, u, v\right\rangle$ and $\left\langle w, \ldots, v_{n}, v_{n+1}\right\rangle$. If the second partial route contains a charging station $z$, that is, $\left\langle w, \ldots, z, z+1, \ldots v_{n+1}\right\rangle$, then the variables should be recalculated for segment $\langle w, \ldots, z+1\rangle$. Similarly, in the case of vertex insertion, in which route $r=\left\langle v_{0}, \ldots, u, v, w, \ldots, v_{n+1}\right\rangle$ is formed by inserting vertex $v$ to route $\left\langle v_{0}, \ldots, u, w, \ldots, v_{n+1}\right\rangle$, recalculation is required for segment $\langle v, \ldots, z+1\rangle$ if the partial route $\left\langle w, \ldots, v_{n}, v_{n+1}\right\rangle$ includes a charging station $z$.

\subsection{Generation of initial solution}

We split the problem into the routing sub-problem and the station location sub-problem and solve them in sequence to generate the initial solution. In the routing sub-problem, the battery capacity of the vehicle is neglected, and no station is considered. The violations of capacity and time windows are also transformed into the penalized costs in the objective function (see Eq. (31)). The generalized cost functions include the routing-related costs in the original objective function and the penalized costs caused by the loading capacity violation $L_{c a p}(S)$ and time window violation $L_{t w}(S)$ The latter two costs are scaled by the penalty factors $\beta_{c a p}$ and $\beta_{t w}$, respectively. In this step, the initial values $\beta_{c a p}^{0}$ and $\beta_{t w}^{0}$ are assigned to the two factors accordingly. 


$$
\begin{aligned}
f_{\text {routing }}(S)= & \sum_{j \in R \cup\left\{0^{0}\right\}} \sum_{k \in K} f_{j}^{k} \cdot c_{e}^{r}+c_{d} \cdot \sum_{k \in K} \tau_{o^{\prime}}^{k} \\
& +\beta_{c a p} \cdot L_{c a p}(\mathrm{~S})+\beta_{t w} \cdot L_{t w}(\mathrm{~S})
\end{aligned}
$$

The solution of the routing problem is generated with $m$ routes, where $m$ is the maximum number of vehicles. Each route is initialized with a seed customer. The seed customer is selected according to the rank of the latest service start time. The remaining customers are then iteratively inserted into the active routes at the position that causes minimal increase in the generalized cost $f_{\text {routing }}(S)$, until no customer is left.

After the initial routes are determined, the feasibility of the solution is further improved in terms of battery capacity constraint. For each infeasible route $r_{k}=\left\langle v_{0}, v_{1}, \ldots, v_{n+1}\right\rangle$, a charging station is iteratively inserted into the route before the first breaking vertex $v_{j}$, which is defined as the vertex that first violates the battery capacity in the route [6]. In general, a vehicle is not assumed to visit a charging station immediately after a visit to another station or depot. Thus, the position where a station should be inserted can be narrowed to a segment of the route. The segment of the route, denoted by set $L_{j}$, is the sequence of vertices that ends with the first breaking vertex $v_{j}$ and starts from the preceding station or depot. For each vertex in the segment $v_{j} \in L_{j}$, an attainable station set is created based on the remaining battery amount $R_{v_{g}}=Q-\Upsilon_{v_{g}}^{\rightarrow}$ at the vertex $v_{g}$, that is, $T_{g}=\left\{w \mid w \in R \wedge d_{v_{g} v_{h}^{\prime}} \cdot \varepsilon \leq R_{v_{g}}\right\}$. The vertex with an empty attainable station set is removed from the set $L_{j}$. Given the attainable set $T_{g}$ of each vertex $v_{g}$ in $L_{j}$, a set that contains all the reachable stations of the segment $L_{j}$ can be generated, that is, $\mathrm{H}\left(L_{j}\right)=\left\{h \mid h \in T_{g} \wedge v_{g} \in L_{j}\right\}$. The vertices in the set are first ranked in ascending order of construction costs, and the vertex $h^{*}$ indexed as $\left.\left|\varpi_{1}^{\rho_{1}}\right| L_{j} \mid\right\rfloor$ is then selected to find the proper station from $\mathrm{H}\left(L_{j}\right)$. Specifically, $\left\lfloor\varpi_{1}^{\rho_{1}}\left|L_{j}\right|\right\rfloor$ is used to introduce randomness, where $\varpi_{1}$ is a random number between 0 and 1 , and $\rho_{1} \geq 1$ is a constant parameter and is equal to 10 in this work [6]. After the selected station is inserted, the feasibility state of route $r_{k}$ is updated. The construction cost of the inserted located station is first recorded as zero to avoid over-counting, because a station may serve multiple routes. For each infeasible route, these steps are repeated until the route is feasible in terms of battery driving limitation.

\subsection{Adaptive variable search component}

In this phase, infeasible solutions are also allowed during the search. However, a new generalized objective function is utilized in this phase because both the routing and location problems are considered. The generalized objective function, defined in Eq. (32), integrates the initial objective function and the penalized costs caused by the loading capacity, time window, and battery capacity violations. As illustrated in Section 4.3, $\beta_{c a p}, \beta_{t w}$, and $\beta_{b a t}$ represent the penalty factors weighting the violations. These factors are initialized with $\left(\beta_{c a p}^{0}, \beta_{t w}^{0}, \beta_{b a t}^{0}\right)$ and adjusted based on the violation status in a predefined number of iteration time $\eta_{p e n}$. In detail, if the loading capacity constraint is violated for $\eta_{p e n}$ iterations, the factor for capacity $\beta_{c a p}$ is multiplied with a factor $\alpha$. In an analogous manner, the factor is divided by a factor $\alpha$, if the capacity constraint is met for $\eta_{p e n}$ iterations. The same mechanism is applied for $\beta_{t w}$ and $\beta_{b a t}$. The factors, $\beta_{c a p}$, and $\beta_{t w}$ are $\beta_{b a t}$ also restricted between the lower bound $\left(\beta_{c a p}^{\min }, \beta_{t w}^{\min }, \beta_{b a t}^{\min }\right)$ and the upper bound $\left(\beta_{c a p}^{\max }, \beta_{t w}^{\max }, \beta_{b a t}^{\max }\right)$ during the process.

$$
f_{A V N S}(S)=f(S)+\beta_{b a t} \cdot L_{c a p}(\mathrm{~S})+\beta_{t w} \cdot L_{t w}(\mathrm{~S})+\beta_{b a t} \cdot L_{b a t}(\mathrm{~S})(3
$$

\subsubsection{Shaking neighborhood structures}

The choice of neighborhood structures is critical for the solution quality. For EV-MCS-LRPTW, five operators are used to define the neighborhood structures in the shaking step. Among the five operators, two are the acknowledged cyclic-exchange and move-neighborhood operators [34], and the other three are problem-specific operators named the station-reconstruct, station-relocate, and station-exchange operators. The cyclic-exchange operator swaps the customer sequences of an arbitrary length among the routes simultaneously. This operator is characterized by the number of routes involved, $\Omega$, and the maximum length of the sequence to exchange, $\Gamma^{\max }$, which is denoted as the number of vertices. In detail, the cyclic-exchange operator transfers a vertex sequence $H_{j_{k}, \Gamma^{k}}^{k}$ with the start point $j_{k}$ and length $\Gamma^{k}$ in route $k$ to route $k+1$ at the former position of sequence $H_{j_{k+1}, \Gamma^{k+1}}^{k+1}$. If the number of existing routes is less than the number of routes to cycle, then the value of $\Omega$ is reduced accordingly. If the maximum length $\Gamma^{\max }$ exceeds the length of route $k$, denoted by $\left|\mathrm{V}_{k}\right|$, then the actual sequence length to exchange $\Gamma^{k}$ is to be adjusted to a random number in the interval $\left[0, \min \left(\Gamma^{\max },\left|\mathrm{V}_{k}\right|\right)\right]$. An example of a cyclic-exchange operator with three routes is shown in Figure 2. The move-neighborhood operator can be regarded as a special form of cyclic-exchange operator. This operator moves the vertex sequence from one route to another, while no vertex is relocated from the second route.

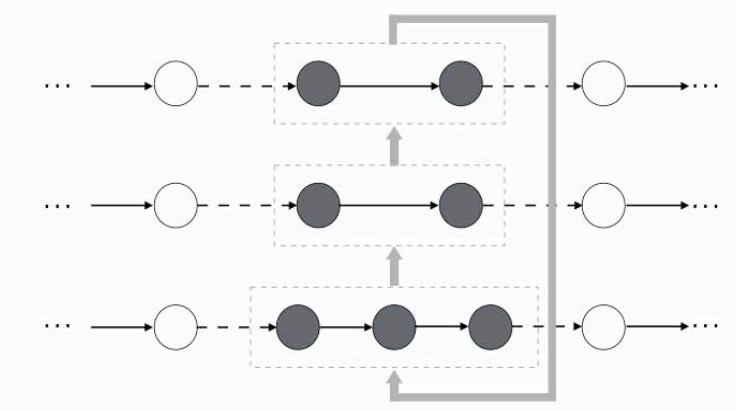

Fig. 2. Graphical illustration of a cyclic-exchange move

The station-reconstruct operator is executed on the charging stations. For a selected route, station-reconstruct first removes all located stations and then inserts certain stations back to make the route feasible in terms of battery capacity. The approach of inserting stations is similar to the generation of the initial solution. The only difference lies in the guidance metrics. The decision in the station-reconstruct 
operator is guided by the generalized objective function of the solution $f_{A V N S}(S)$, instead of the construction cost in the generation of the initial solution. Each station $h$ in the reachable set of the route segment $\mathrm{H}\left(L_{j}\right)$ is sorted in ascending order of the objective function value of the resulting solution $f_{A V N S}(S \leftarrow h)$, in which station is inserted. The station indexed as $\left.\left|\varpi_{2}^{\rho_{2}}\right| H\left(L_{j}\right) \mid\right\rfloor$ is then selected, where $\varpi_{2}$ and $\rho_{2}$ are defined the same as $\varpi_{1}$ and $\rho_{1}$, respectively. After the selected station is located, the feasibility state of the route is updated. For the selected route, station-reconstruct repeats the preceding steps until the route becomes feasible in terms of battery driving limitation.

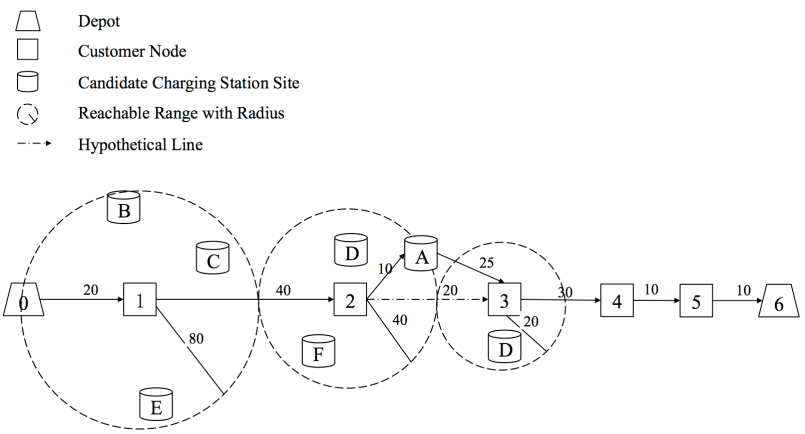

Fig. 3. Graphical illustration of the neighboring station set

Station-relocate replaces a current station with an unlocated one in its neighboring station set. The neighboring station set of a located station is the set of stations that can replace the located one without affecting the feasibility of the solution regarding battery capacity. We provide a simple example in Figure 3 to better illustrate the neighboring set. The neighboring station set of the located station A can be determined as follows: Station A is removed from the route, and the separated vertices, $v_{2}$ and $v_{3}$, are directly connected. The first breaking vertex in the resulting route, which is vertex $v_{4}$, is found. The route segment before the first breaking vertex after the depot or a preceding station, which is $\left\{v_{1}, v_{2}, v_{3}\right\}$, is identified, and the reachable set of the segment, which is $\{A, B, C, D, E, F, G\}$, is determined. The neighboring station of station $\mathrm{A}$ is the reachable set of the route segment excluding itself, that is, $N(A)=\{B, C, D, E, F, G\}$. The neighboring set can further be divided into two sets $N_{\text {open }}(A)$ and $N_{\text {unopen }}(A)$ based on whether or not the stations in the neighboring set are located. After obtaining un-located stations in the neighboring set $N_{\text {unopen }}(A)$, the station-relocate operator ranks the stations in the set $N_{\text {unopen }}(A)$ in ascending order of objective function value of the resulting solution $f_{A V N S}\left(S^{\prime}\right)$ and replaces the station with the one indexed by $\left\lfloor\varpi_{3}^{\rho_{3}}\left|N_{\text {unopen }}(w)\right|\right\rfloor$, where $\varpi_{3}$ and $\rho_{3}$ are defined the same as $\varpi_{1}$ and $\rho_{1}$, respectively. The station-exchange operator substitutes the current station with a located one in its neighboring station set, and the implementation is the same as in the station-relocate operator, but with set $N_{\text {open }}(A)$.

Table 1 presents the neighborhood structures based on the five operators within the shaking step of AVNS. The first nine structures consist of six move-neighborhoods, followed by one station-reconstruct, one station-relocate, and one station-exchange. The three sets of neighborhood structures that comprise six cyclic-exchanges, one stationreconstruct, one station-relocate, and one station-exchange are presented from rows 10 to 18,19 to 27 , and 28 to 36 . The number of routes involved $\Omega$ is not relevant for station-relocate and station-exchange but stands for the involved routes in station-reconstruct. The maximum length to exchange $\Gamma^{\max }$ in station-relocate and station-exchange represents the maximum number of charging stations to be relocated or exchanged, but it is irrelevant to stationreconstruct. Other rules of $\Omega$ and $\Gamma^{\max }$ are also applicable to problem-specific operators.

Table 1. Parameters of the four real-world networks

\begin{tabular}{|c|c|c|c|}
\hline$\kappa$ & Type & $\Omega$ & $\Gamma^{\max }$ \\
\hline 1 & Move-neighborhood & 2 & 1 \\
\hline 2 & Move-neighborhood & 2 & 2 \\
\hline 3 & Move-neighborhood & 2 & 3 \\
\hline 4 & Move-neighborhood & 2 & 4 \\
\hline 5 & Move-neighborhood & 2 & 5 \\
\hline 6 & Move-neighborhood & 2 & 6 \\
\hline 7 & Station-reconstruct & 1 & - \\
\hline 8 & Station-relocate & - & 1 \\
\hline 9 & Station-exchange & - & 1 \\
\hline 10 & Cyclic-exchange & 2 & 1 \\
\hline 11 & Cyclic-exchange & 2 & 2 \\
\hline 12 & Cyclic-exchange & 2 & 3 \\
\hline 13 & Cyclic-exchange & 2 & 4 \\
\hline 14 & Cyclic-exchange & 2 & 5 \\
\hline 15 & Cyclic-exchange & 2 & 6 \\
\hline 16 & Station-reconstruct & 2 & - \\
\hline 17 & Station-relocate & - & 2 \\
\hline 18 & Station-exchange & - & 2 \\
\hline 19 & Cyclic-exchange & 3 & 1 \\
\hline 20 & Cyclic-exchange & 3 & 2 \\
\hline 21 & Cyclic-exchange & 3 & 3 \\
\hline 22 & Cyclic-exchange & 3 & 4 \\
\hline 23 & Cyclic-exchange & 3 & 5 \\
\hline 24 & Cyclic-exchange & 3 & 6 \\
\hline 25 & Station-reconstruct & 3 & - \\
\hline 26 & Station-relocate & - & 3 \\
\hline 27 & Station-exchange & - & 2 \\
\hline 28 & Cyclic-exchange & 4 & 1 \\
\hline 29 & Cyclic-exchange & 4 & 2 \\
\hline 30 & Cyclic-exchange & 4 & 3 \\
\hline 31 & Cyclic-exchange & 4 & 4 \\
\hline 32 & Cyclic-exchange & 4 & 5 \\
\hline 33 & Cyclic-exchange & 4 & 6 \\
\hline 34 & Station-reconstruct & 4 & \\
\hline 35 & Station-relocate & - & 4 \\
\hline 36 & Station-exchange & - & 2 \\
\hline
\end{tabular}

\subsubsection{Adaptive shaking}

The AVNS in this work applies selection approaches in biasing the route, vertex sequence, and station selection involved in the shaking step to find promising solutions. The located station selection methods are newly designed for the problem, and the route and vertex sequence selection methods are similar to those in the VRPIS studied by Schneider et al. [29].

Route selection methods: Five selection methods are adopted to determine the first route in the subset of $\Omega$ routes that is involved in cyclic-exchange and stationreconstruct. The selection is performed in an iterative way; 
that is, after selecting the first route, the remaining routes are iteratively selected from the routes that are spatially closer than a predefined threshold to the recently selected route. If no route is available in the confined area, the next route is to be randomly selected among all the routes. The selection mechanisms adopted to determine the first route are as follows:

1) Random: The probability of selecting each route has no difference.

2) Route length: The probability of selecting each route is proportional to the total length of the route.

3) Route length per demand unit: The probability of selecting each route is proportional to the ratio between total length and total demand.

4) Station density: The probability of selecting each route is proportional to the ratio of the number of stations to the number of total customers on the route.

5) Station detour: The probability of selecting each route is proportional to the total detour caused by the charging station of the route.

Vertex sequence selection methods: After the routes involved in cyclic-exchange are selected, the vertex sequence in each route is determined according to the following three mechanisms:

1) Random: The probability of selecting each vertex sequence has no difference.

2) Distance to the next route: The probability of selecting each vertex sequence is inversely proportional to the distance from the sequence to the route where it is going to be inserted. The distance is measured by summing up the distance of each vertex in the sequence to the center of the target route.

3) Distance to the neighboring vertex sequence: The probability of selecting each vertex sequence is proportional to the distance of the sequence to its surrounding vertices. The distance is calculated as the sum of the distance between the first vertex and its predecessor and that between the last vertex and its successor.

Station selection methods: Four selection approaches are proposed to select the located station. In particular, methods 1,2 , and 3 are used to select the to-be-replaced stations in the station-relocate operator, and methods 1,2 , and 4 are used to determine the stations in the station-exchange operator.

1) Random: The probability of selecting each station has no difference.

2) Number of related routes: The probability of selecting each station is proportional to the number of routes in the station services.

3) Number of neighboring un-located stations. The probability of selecting each station is proportional to the number of un-located stations in the neighboring station set.

4) Number of neighboring located stations. The probability of selecting each station is proportional to the number of located stations in the neighboring station set.

\subsubsection{Adaptive mechanism}

Given that the performance of each selection method differs across different problems, we apply the roulette wheel selection method to bias the selection of these methods based on probabilities [35]. As in ALNS, an adaptive mechanism is utilized in AVNS to assess the importance of selection methods by updating their probabilities. Considering a total of $s$ selection methods, $i=1,2, \ldots, s$, each method is associated with a weight $\omega_{i}$, and the probability of selecting the method $i$ is given by $\omega_{i} / \sum_{j=1}^{s} \omega_{j}$. The weight of each selection method $\omega_{i}$ is initialized with an equal weight at the beginning and updated every $\eta_{A V N S}$ AVNS iteration based on its performance. The performance of each method is measured by a scoring system, which assigns a score of nine to the method when a new overall best solution is found by the method, a score of three when the current solution is improved, and a score of one when the solution is worse than the current solution but is accepted by the acceptance criterion. Given the current score of method $i$, denoted by $s_{i}$, and the number of times it has been selected since the last weight update, denoted by $z_{i}$, the new weight is then recalculated as $\omega_{i} \cdot\left(1-\rho_{a d p}\right)+\rho_{a d p} \cdot\left(s_{i} / z_{i}\right) . \rho_{a d p} \in[0,1]$ is a parameter that controls the adaptive behavior, and $s_{i}, z_{i}$ are reset to zero after each weight update.

\subsection{Tabu search component}

The TS algorithm is applied to improve the routing plans in the solution $S^{\prime}$ generated from the AVNS phase. In every iteration, four operators are executed on the arc in the list of generator arcs to develop the neighborhood of the TS. The four operators are composed of three well-known operators, 2-opt*, relocate, exchange, and one problem-specific operator, StationInRe [22,36,37] . As is commonly done in the content of VRP, the TS algorithm regards a move as superior if the move can reduce the number of employed vehicles or has a better function value calculated with Eq. (42). In detail, the 2-opt* operator modifies the famous 2-opt to avoid the reversal of route directions. This operator is implemented for inter-route moves and allows the removal and insertion of the arc that includes a station visit. The relocate operator removes one vertex and inserts it into a different position on the same route or another route. The exchange operator switches the positions of the two vertices. Both relocate and exchange are applied for intra- and interroute moves. However, the swapping of a station with a customer or another station is defined only for relocate. StationReIn is introduced to solve E-VRPTW, and it performs insertion and removals of charging stations.

The TS algorithm forbids the reinsertion of an arc into specific parts of the solution for a predefined number of iterations denoted by $\varsigma$, which is randomly drawn from $\left[\varsigma_{\min }, \varsigma_{\max }\right]$. Given that a visit to a station has a great effect on the battery level and time window obedience, a tabu attribute characterized by four elements $(l, k, v, w)$ is defined to prevent violations caused by reinsertion. The tabu attribute is used to prevent arc $\imath$ from being inserted into route $r_{k}$ between $v$ and $w$, where $v$ and $w$ denote stations or depot. Through this process, an arc can be reinserted into a different part of the route. During the procedure, if a feasible new best solution is found, then the tabu status of a move is favored. After the predefined number of iteration $\eta_{T S}$, the TS process is terminated. The best solution found during TS is stored in solution $S$ " as output.

\subsection{Improvement procedure}

Similar to the work of Yang et al., the current study enhances the solution found by the former algorithm through 
an improvement procedure [6]. For all the located stations in each route $r_{k}$, the procedure first selects the charging stations in order and then removes the station from the route. If the removal of a station results in a battery capacity violation, the route is then split at the point with the least cost value. The cost value is calculated by $c_{e}^{r^{*}} \cdot \mathcal{E} \cdot\left(d_{i, o^{\prime}}+d_{o, i+1}-d_{i, i+1}\right)+c_{d} \cdot\left(t_{i, o^{\prime}}+t_{o, i+1}-t_{i, i+1}\right)$, where $i$ denotes the split point, $o, o^{\prime}$ the instances of depot, and $c_{e}^{r^{*}}$ the electricity price in depot. The procedure ends when the number of vehicles approaches the maximum limitation or the solution cannot be improved any further. The solution produced, denoted by $S$ '", is compared with the current best solution $S$ and evaluated by the acceptance mechanism illustrated below. If the solution $S^{\prime \prime}$ is accepted, then $S^{\prime \prime \prime}$ replaces $S$, and $\kappa$ is reset to 1 .

\subsection{Acceptance mechanism}

Aside from always favoring an improving solution, the AVNS/TS algorithm also accepts a new deteriorating solution if it passes the acceptance criterion [38]. The acceptance criterion is developed based on SA and allows the unsatisfying solution to be a new-current solution with a certain probability $e^{-\left(f_{A V N S}\left(S^{\prime \prime \prime)}\right)-f_{A V N S}(S)\right) / T}$. The value of the probability is based on the difference between the new and current solutions, that is, $f_{A V N S}\left(S^{\prime \prime \prime}\right)-f_{A V N S}(S)$, and temperature $T$. Temperature $T$ is initialized with $T_{0}$ and updated using $T_{n}=\vartheta \cdot T_{n-1}$ after each iteration. If no improving solution emerges in a predefined number of iterations denoted by $\eta_{n o n}$, then the current solution is reset to the best solution found so far. The value of $T$ is set to $T_{0}$ after $\eta_{\text {reset }}$ solution resets to diversify the solution.

\section{Experimental studies}

The performance of AVNS/TS on different sizes of instances is explored in terms of solution quality and computation time in this section. First, the parameter tuning is illustrated in Section 5.1, followed by the generation of instances in Section 5.2. Subsequently, the solution quality and effectiveness of the AVNS/TS algorithm are assessed with small- and large-scale instances in Section 5.3.

\subsection{Experimental environment and parameter setting}

The experiments are conducted on a desktop computer with an Inter Core i5 processor at $2.67 \mathrm{GHz}$, with $4 \mathrm{~GB}$ of RAM and Windows 7 Professional. The AVNS/TS algorithm is implemented as a single-thread code in Java. Ten reasonably large instances are randomly generated to tune the parameters. The initial values of the parameters found during the development of our algorithm are input as basis for the tuning. One parameter is adjusted, while the others are kept

Table 2 summarizes the parameter settings for the initial penalty factors $\beta_{c a p}^{0}, \beta_{t w}^{0}, \beta_{b a t}^{0}$, the bounds $\beta_{c a p}^{\min }, \beta_{t w}^{\min }, \beta_{b a t}^{\min }$ and $\beta_{c a p}^{\max }, \beta_{t w}^{\max }, \beta_{b a t}^{\max }$, the penalty update factor $\alpha$, and the number of penalty update iterations $\eta_{p e n}$. For the algorithm, the number of iterations after which the probabilities are updated is $\eta_{a d p}$. The parameter that weighs the old and new scores in the adaptive mechanism is $\rho_{\text {adp }}$. The minimal and maximum tabu tenures are $\varsigma_{\min }$ and $\varsigma_{\max }$, respectively. The number of TS iterations is $\eta_{T S}$. The initial temperature parameter value is $T_{0}$. The cooling rate is $\vartheta$. The number of iterations after which the current solution is reset to the global best solution is $\eta_{\text {non }}$. The number of solution resets after which the temperature parameter is reset is $\eta_{\text {reset }}$.

fixed. The best value of each parameter is determined after 20 runs on the generated test instances.

Table 2. Parameters of the parameters in AVNS/TS

\begin{tabular}{|c|c|c|c|c|c|}
\hline \multicolumn{2}{|l|}{ Penalties } & \multicolumn{2}{|c|}{ AVNS/ TS } & \multicolumn{2}{|l|}{ SA } \\
\hline$\beta_{c a p}^{0}, \beta_{t w}^{0}, \beta_{b a t}^{0}$ & 10 & $\rho_{a d p}$ & 0.3 & $T_{0}$ & 50 \\
\hline$\beta_{c a p}^{\min }, \beta_{t w}^{\min }, \beta_{b a t}^{\min }$ & 0.5 & $\eta_{a d p}$ & 20 & $\vartheta$ & 0.995 \\
\hline$\beta_{c a p}^{\max }, \beta_{t w}^{\max }, \beta_{b a t}^{\max }$ & 1000 & $\varsigma_{\min }$ & 15 & $\eta_{\text {non }}$ & 25 \\
\hline$\alpha$ & 1.5 & $\varsigma_{\max }$ & 40 & $\eta_{\text {reset }}$ & 4 \\
\hline$\eta_{p e n}$ & 2 & $\eta_{T S}$ & 100 & & \\
\hline
\end{tabular}

We set the maximal number of AVNS/TS iterations to $\eta=800$ for instances with less than 100 customers and $\eta=800 /(0.01 \cdot|C|)^{1.5}$ for instances with more customers to achieve a good trade-off between run time and solution quality.

\subsection{Generation of test instances}

The instances for EV-MCS-LRPTW are generated based on the instances introduced in the pollution-routing problem (PRP) research of Demir et al., which consist of nine sets of instances with different sizes [39]. Specifically, we referred to their instances for network, customer demand, time windows, vehicle capacity, and driver wages. The speed of the vehicle is set to $90 \mathrm{~km} / \mathrm{h}$, the maximum speed in their research, to guarantee that all instances have at least one feasible solution in terms of time windows. The battery capacity of the vehicle is set to $80 \mathrm{kWh}$, as in the work of Davis et al. [40].

The candidate charging station sites are randomly selected from the customer vertices. The number of candidate charging stations is determined as follows, according to the number of customers: If the number of customers is less than 50, then all the customer sites are assumed to be candidate station sites. Otherwise, $20 \%$ of the customers are assumed to be candidate station sites. One charging station is assumed to be located at the depot. Without loss of generality, four types of charging infrastructure are considered to be options in each candidate site, which are slow charging station (SCS), fast charging station (FCS), super-fast charging station (SFCS), and BSS. The construction cost comprises the set-up cost and rental cost in this case. The charging rate, the cost components including initial expenditure, and the station lifetime of Level II AC, Level III DC, and "super-fast" DC charging station in the work of Schroeder et al. are used for the charging rate, set-up cost, and lifetime of SCS, FCS, and SFCS in this work, respectively, based on the exchange rate of EUR to USD as 1.10[5]. The swap time and equipment cost of BSS are assumed to be $10 \mathrm{~min}$ and 500,000 USD, respectively [7,21]. The set-up cost input in the model is the cost that is amortized to one day based on the lifetime. The average rental cost of land is assumed to be $2.19 \mathrm{USD} / \mathrm{m}^{2}$ per day with a random fluctuation within $10 \%$ for different locations [41]. The areas of SCS, FCS, and SFCS are 
assumed to be $25 \mathrm{~m}^{2}$, and that of BSS is assumed to be twice that figure because of the automated equipment [32]. For the cost of back-up batteries at BSS, an apportioned cost by travel distance, called battery replacement cost, is utilized, instead of the total cost [20]. The electricity price at SCS, FCS, and SFCS is assumed to be the weighted average of the price at the peak period $(0.5 \mathrm{USD} / \mathrm{kWh}$ for $2 \mathrm{pm}-8 \mathrm{pm})$ and the price at the mid-peak period $(0.2 \mathrm{USD} / \mathrm{kWh}$ for $7 \mathrm{am}-2$ $\mathrm{pm}$ and $8 \mathrm{pm}-10 \mathrm{pm})$, and that at the BSS and depot is the price of the off-peak period, $0.15 \mathrm{USD} / \mathrm{kWh}$ [42]. Table 3 summarizes the data.

\subsection{Experiments on EV-MCS-LRPTW instances}

In the numerical tests, the performance of the algorithm is first assessed on small-scale instances by comparing its results with those of the CPLEX Solver 12.2, and then it is evaluated with large-scale instances.

Table 3. Data of EV-MCS-LRPTW instances

\begin{tabular}{|c|c|c|c|}
\hline Description & Value & Description & Value \\
\hline Loading capacity & 3650 & Set-up cost of BSS (\$) & 500000 \\
\hline Speed $(\mathrm{km} / \mathrm{h})$ & 90 & Area of SCS, FCS, and SFCS $\left(\mathrm{m}^{2}\right)$ & 25 \\
\hline Battery capacity $(\mathrm{kWh})$ & 80 & Area of BSS $\left(\mathrm{m}^{2}\right)$ & 50 \\
\hline Stated range $(\mathrm{km})$ & 161 & Average rental cost $\left(\$ / \mathrm{m}^{2} /\right.$ day $)$ & 2.19 \\
\hline Speed $(\mathrm{km} / \mathrm{h})$ & 90 & Station lifetime of SCS (years) & 15 \\
\hline Charging rate at $\mathrm{SCS}(\mathrm{kWh} / \mathrm{min})$ & 0.12 & Station lifetime of FCS (years) & 15 \\
\hline Charging rate at FCS $(\mathrm{kWh} / \mathrm{min})$ & 1.05 & Station lifetime of SFCS (years) & 10 \\
\hline Charging rate at SFCS $(\mathrm{kWh} / \mathrm{min})$ & 4.00 & Station lifetime of BSS (years) & 20 \\
\hline Time of battery swap at BSS (min) & 10 & Battery replacement cost $(\$ / \mathrm{km})$ & 0.20 \\
\hline Set-up cost of SCS $(\$)$ & 6600 & Driver wages $(\$ / h)$ & 13.14 \\
\hline Set-up cost of FCS $(\$)$ & 60500 & Average electricity cost at SFCS, SCS, FCS $(\$ / \mathrm{kWh})$ & 0.32 \\
\hline Set-up cost of SFCS (\$) & 126500 & Electricity cost at BSS and depot $(\$ / \mathrm{kWh})$ & 0.15 \\
\hline
\end{tabular}

Table 4. Comparison of results obtained with CPLEX and AVNS/TS on the small-scale EV-MCS-LRPTW instances

\begin{tabular}{l|l|l|l|l|l|l|l|l}
\hline \multicolumn{9}{l}{ Input } \\
\hline Instance & $\mid \mathbf{C}$ & $|\mathbf{R}|$ & $|\mathbf{K}|$ & Best & Time (s) & Best & Time (s) & Gap (\%) \\
\hline UK50_01_N6 & 6 & 6 & 2 & 199.16 & 49.32 & 199.16 & 0.44 & 0 \\
UK75_05_N6 & 6 & 6 & 2 & 68.19 & 63.04 & 68.19 & 0.42 & 0 \\
UK100_18_N6 & 6 & 6 & 2 & 194.31 & 134.39 & 194.31 & 0.39 & 0 \\
UK50_03_N8 & 8 & 8 & 3 & 210.10 & 1549.42 & 210.10 & 0.81 & 0 \\
UK75_11_N8 & 8 & 8 & 3 & 226.20 & 1004.79 & 226.20 & 0.74 & 0 \\
UK100_15_N8 & 8 & 8 & 3 & 387.96 & 959.48 & 387.96 & 0.59 & 0 \\
UK50_05_N10 & 10 & 10 & 3 & 394.54 & 6503.1 & 394.54 & 1.39 & 0 \\
UK75_12_N10 & 10 & 10 & 3 & - & 7200 & 403.11 & 1.15 & - \\
UK100_18_N10 & 10 & 10 & 3 & - & 7200 & 338.89 & 1.46 & - \\
\hline
\end{tabular}

Tab. 5. Results of results AVNS/TS on the large-scale EV-MCS-LRPTW instances

\begin{tabular}{|c|c|c|c|c|c|c|c|c|c|}
\hline \multicolumn{4}{|l|}{ Input } & \multicolumn{6}{|c|}{ Result } \\
\hline Instance & $|\mathbf{C}|$ & $|\mathbf{R}|$ & $|\mathbf{K}|$ & SCS & FCS & SFCS & BSS & Best & Time (min) \\
\hline UK50_01 & 50 & 50 & 10 & 1 & 2 & 1 & 0 & 838.88 & 10.56 \\
\hline UK50_07 & 50 & 50 & 10 & 1 & 1 & 0 & 0 & 541.29 & 7.51 \\
\hline UK50_10 & 50 & 50 & 10 & 1 & 2 & 1 & 0 & 906.05 & 16.27 \\
\hline UK50_14 & 50 & 50 & 10 & 1 & 2 & 1 & 0 & 889.90 & 13.00 \\
\hline UK75_03 & 75 & 15 & 15 & 1 & 3 & 0 & 0 & 1020.01 & 21.53 \\
\hline UK75_05 & 75 & 15 & 16 & 1 & 2 & 1 & 0 & 1061.73 & 34.92 \\
\hline UK75_11 & 75 & 15 & 16 & 1 & 2 & 0 & 0 & 794.56 & 16.84 \\
\hline UK75_17 & 75 & 15 & 16 & 1 & 2 & 1 & 0 & 1084.41 & 30.56 \\
\hline UK100_07 & 100 & 20 & 19 & 1 & 3 & 1 & 0 & 1328.59 & 57.47 \\
\hline UK100_09 & 100 & 20 & 20 & 1 & 1 & 1 & 0 & 1055.17 & 48.90 \\
\hline UK100_12 & 100 & 20 & 19 & 1 & 2 & 1 & 0 & 1199.35 & 50.34 \\
\hline UK100_19 & 100 & 20 & 20 & 1 & 3 & 2 & 0 & 1598.84 & 71.22 \\
\hline UK150_01 & 150 & 30 & 30 & 1 & 2 & 1 & 0 & 1542.47 & 105.06 \\
\hline UK150_05 & 150 & 30 & 30 & 1 & 2 & 1 & 0 & 1587.59 & 107.30 \\
\hline UK150_13 & 150 & 30 & 29 & 1 & 3 & 0 & 0 & 1649.29 & 84.45 \\
\hline UK150_19 & 150 & 30 & 30 & 1 & 2 & 2 & 0 & 1941.96 & 146.07 \\
\hline UK200_03 & 200 & 40 & 42 & 1 & 3 & 0 & 0 & 2003.44 & 113.41 \\
\hline
\end{tabular}




\begin{tabular}{l|l|l|l|l|l|l|l|l|l} 
UK200_07 & 200 & 40 & 40 & 1 & 2 & 1 & 0 & 2002.45 & 210.83 \\
UK200_10 & 200 & 40 & 42 & 1 & 1 & 1 & 0 & 2037.46 & 171.25 \\
\hline UK200_16 & 200 & 40 & 40 & 1 & 2 & 1 & 0 & 2046.07 & 193.24 \\
\hline
\end{tabular}

The quality and run time of AVNS/TS on small instances is tested using several instances. The instances are generated by randomly selecting $|C|$ customers from the given instances. For example, UK50_01_N6 represents the instance in which six customers are selected from 50 customers in PRP instances. Both the AVNS/TS algorithm and the CPLEX solver are applied to solve the generated instances. The results are summarized in Table 4.

For clarity, columns 1 to 4 describe the basic structures of instances, where the number of customer number, candidate station sites, and vehicles are presented sequentially. The results and computing time are listed in columns 5 and 6 , respectively. Columns 7 to 9 show the computational result of the AVNS/TS algorithm, computing time, and gap between the results using AVNS/TS and the CPLEX. As is commonly done, a time limit of 7,200s (2h) is set for all the instances. If the CPLEX solver fails to find the optimal solution for an instance, we use the symbol "-" to indicate its result. From the computing results, the AVNS/TS algorithm can effectively find the optimal solution.

Subsequently, the AVNS/TS is applied to solve largescale instances. Table 5 presents the results of 20 instances with objective function values and computation times. In detail, columns 1 to 4 depict the feature of the instances. Columns 5 to 8 show the result of the charging station strategy. For each instance, the algorithm is executed five times, from which the best solution is recorded in column 9, and the related run time in minute is presented in column 10.

The computation results imply that AVNS/TS can solve large-scale instances within reasonable time. The optimal solutions of several instances indicate that multiple types of charging stations should be considered to reach the minimum cost. The results also imply that SCS, with a minimal construction cost among the four types of stations, is suitable for the central depot, which is reasonable from the cost perspective. Meanwhile, a combination of FCS and SFCS is more suitable in most cases. The BSS type is not selected for any of the 20 instances mostly because its construction cost is much higher than those of FCS and SFCS.

\section{Conclusions}

This paper introduces EV-MCS-LRPTW, an extension of LRP, to optimize the routing of EVs and the strategy of charging stations under the constraints of loading capacity, battery capacity, and time windows. Contrary to existing research on EVs, this problem considers the possibility of using different types of charging infrastructure and optimizes the type selection of infrastructures along with the station location decision and routing plan. This problem is highly relevant in the context of EVs because charging stations are critical to EVs. The optimal selection of charging infrastructure can save money for logistic companies in the long run because each charging technology has its pros and cons in terms of charging speed, economic investment, and practical requirement.

We developed a hybrid heuristic that combines AVNS with the TS algorithm for intensification to address the problem. A number of instances with realistic data were designed to validate the algorithm in the experimental studies. The computational results indicate that the algorithm can effectively reach nearly optimal solutions for small-scale instances, in contrast to the CPLEX solver. The algorithm also provided convincing results in moderate run time for large-scale instances. The results on realistic-sized instances demonstrate that incorporating multiple types of charging infrastructure in the station strategy and routing plan can result in a better solution than considering a single charging infrastructure under the constraints of time windows.

In future, the problem can be extended to include multiple depots or consider a mixed fleet with EVs and ICVs. The existence of public charging stations can also be incorporated into the problem. The work can be extended by further considering specific characteristics of EVs.

\section{Acknowledgements}

This work was supported by the National Natural Science Foundation of China under the project No.71271137, and the Natural Science Foundation of Shanghai under the project No.12ZR1415100.

\section{References}

1. Eggers, F., Eggers, F., "Where have all the flowers gone? forecasting green trends in the automobile industry with a choice-based conjoint adoption model". Technological Forecasting \& Social Change, 78(1), 2011, pp.51-62.

2. National Renewable Energy Laboratory, "Project Startup: Evaluating the Performance of Frito Lay's Electric Delivery Trucks.” 2014. Web. 3 Dec. 2015. <http://www.nrel.gov/docs/fy14osti/61455.pdf>.

3. Heineken International "Case Studies: Europe's Largest Electric Truck Will Drive down Emissions.” 2014. Web. 2 Dec. 2015. $<$ http://sustainabilityreport.heineken.com/ Reducing-CO2emissions/Case-studies/Europes-largest-electric-truck-will-drive-downemissions/index.htm $>$.

4. DHL International, "Deutsche Post DHL Fleet of Alternative Vehicles Continues to Grow." 2014. Web. 2 Dec. 2015. $<$ http://www.dhl.com/en/press/releases/releases_2014/group/dp_dhl_fle et_of_alternative_vehicles_continues_to_grow.httml $>$.
5. Wang, Y. W., Lin, C. C., "Locating multiple types of recharging stations for battery-powered electric vehicle transport". Transportation Research Part E: Logistics \& Transportation Review, 58, 2013, pp. 7687.

6. Yang, J., Sun, H., "Battery swap station location-routing problem with capacitated electric vehicles". Computers \& Operations Research, 55, 2015, pp.217-232.

7. Schneider, M., Stenger, A., Goeke, D., "The electric vehicle-routing problem with time windows and recharging stations". Transportation Science, 48(4), 2014, pp.500-520.

8. Nagy, G., Salhi, S., "Location-routing: Issues, models and methods". European Journal of Operational Research, 177(2), 2007, pp. 649-672

9.Maranzana, F. E., "On the location of supply points to minimize transport costs". Operational Research Society, 15(3), 1964, pp. 261270 . 
10. Boventer, E., "The relationship between transportation costs and location rent in transportation problems". Journal of Regional Science, 3(2), 1961, pp. 27-40.

11. Perboli, G., Tadei, R., Vigo, D., "The two-echelon capacitated vehicle routing problem: Models and math-based heuristics". Transportation Science, 45(3), 2011, pp. 364-380.

12. Govindan, K., Jafarian, A., Khodaverdi, R., Devika, K., "Twoechelon multiple-vehicle location-routing problem with time windows for optimization of sustainable supply chain network of perishable food". International Journal of Production Economics, 152, 2014, pp. 9-28.

13. Sajjadi, S. R., Cheraghi, S. H., "Multi-products location-routing problem integrated with inventory under stochastic demand". International Journal of Industrial and Systems Engineering, 7(4), 2011, pp. 454-476.

14. Guerrero, W. J., Prodhon, C., Velasco, N., Amaya, C. A., "Hybrid heuristic for the inventory location-routing problem with deterministic demand". International Journal of Production Economics, 146(1), 2013, pp. 359-370

15. Semet, F., Taillard, E., "Solving real-life vehicle routing problems efficiently using tabu search". Annals of Operations research, 41(4), 1993, pp. 469-488

16. Zarandi, M., Hemmati, A., Davari, S., "The multi-depot capacitated location-routing problem with fuzzy travel times". Expert Systems with Applications, 38(8), 2011, pp. 10075-10084.

17. Zarandi, M., Hemmati, A., Davari, S., Turksen, I., "Capacitated location- routing problem with time windows under uncertainty". Knowledge-Based Systems, 37, 2013, pp. 480-489.

18. Prodhon, C., Prins, C., "A survey of recent research on locationrouting problems". European Journal of Operational Research, 238(1), 2014, pp. 1-17.

19. Drexl, M., Schneider, M., "A survey of variants and extensions of the location-routing problem". European Journal of Operational Research, 241(2), 2015, pp. 283-308.

20. Jokar, A., Sahraeian, R., "A heuristic based approach to solve a capacitated location-routing problem". Journal of Management and Sustainability, 2(2), 2012, pp. 219.

21. Hemmelmayr, V. C., Cordeau, J. F., Crainic, T. G., "An adaptive large neighborhood search heuristic for two-echelon vehicle routing problems arising in city logistics". Computers \& Operations Research, 39(12), 2012, pp. 3215-3228.

22. Perl, J., Daskin, M. S., "A warehouse location-routing problem". Transportation Research Part B: Methodological, 19(5), 1985, pp. 381396.

23. Conrad, R. G., Figliozzi, M. A., "The recharging vehicle routing problem". Proceedings Industrial Engineering Research Conference. 2011.

24. Erdoğan, S., Miller-Hooks, E., "A green vehicle routing problem". Transportation Research Part E: Logistics and Transportation Review, 48(1), 2012, pp. 100-114.

25. Felipe, Á., Ortuño, M. T., Righini, G., Tirado, G., "A heuristic approach for the green vehicle routing problem with multiple technologies and partial recharges". Transportation Research Part E: Logistics and Transportation Review, 71, 2014, pp.111-128.
26. Goeke, D., Schneider, M., "Routing a mixed fleet of electric and conventional vehicles". European Journal of Operational Research, 245(1), 2015, pp. 81-99.

27. Melechovský, J., Prins, C., Calvo, R. W., "A metaheuristic to solve a location-routing problem with non-linear costs". Journal of Heuristics, 11(5-6), 2005, pp. 375-391.

28. Stenger, A., Vigo, D., Enz, S., Schwind, M., “An adaptive variable neighborhood search algorithm for a vehicle routing problem arising in small package shipping". Transportation Science, 47(1), 2013, pp. 6480 .

29. Schneider, M., Stenger, A., Hof, J., "An adaptive VNS algorithm for vehicle routing problems with intermediate stops". OR Spectrum, 37(2), 2015, pp. 353-387.

30. Glover, F., Laguna, M., Tabu Search. Boston: Kluwer Academic, 1997. Print

31. Psaraftis, H. N., "K-interchange procedures for local search in a precedence-constrained routing problem". European Journal of Operational Research, 13(4), 1983, pp. 391-402.

32. Nagata, Y., Bräysy, O., Dullaert, W., "A penalty-based edge assembly memetic algorithm for the vehicle routing problem with time windows". Computers \& Operations Research, 37(4), 2010, pp. 724737.

33. Schneider, M., Sand, B., Stenger, A., "A note on the time travel approach for handling time windows in vehicle routing problems". Computers \& Operations Research, 40(10), 2013, pp. 2564-2568.

34. Thompson, P. M., Orlin, J. B., "The theory of cyclic transfers". Working paper 1989, Operations Research Center, Massachusetts Institute of Technology. Web. 3 Dec. 2015.

35. Pisinger, D., Ropke, S., "A general heuristic for vehicle routing problems". Computers \& Operations Research, 34(8), 2007, pp. 24032435.

36. Toth, P., Vigo, D., "The granular tabu search and its application to the vehicle-routing problem". Informs Journal on Computing, 15(4), 2003, pp. 333-346.

37. Savelsbergh, M. W. P., "Local search in routing problems with time windows". Annals of Operations Research, 4(1), 1985, pp. 285-305.

38. Kirkpatrick, S., Gelatt, C. D., Vecchi, M. P., "Optimization by simulated annealing". Science, 220, 1983, pp. 671-680.

39. Demir, E., Bektaș, T., Laporte, G., "An adaptive large neighborhood search heuristic for the pollution-routing problem". European Journal of Operational Research, 223(2), 2012, pp. 346-359.

40. Davis, B. A., Figliozzi, M. A., "A methodology to evaluate the competitiveness of electric delivery trucks". Transportation Research Part E: Logistics and Transportation Review, 49(1), 2013, pp. 8-23.

41. Sadeghi-Barzani, P., Rajabi-Ghahnavieh, A., Kazemi-Karegar, H., "Optimal fast charging station placing and sizing". Applied Energy, 125, 2014, pp. 289-299.

42. Zheng, Y., Dong, Z. Y., Xu, Y., Meng, K., Zhao, J. H., Qiu, J., "Electric vehicle battery charging/swap stations in distribution systems: comparison study and optimal planning". Power Systems, IEEE Transactions on Power Systems, 29(1), 2014, pp. 221-229. 